\title{
Most Strange Dibaryon from Lattice QCD
}

\author{
Shinya Gongyo, ${ }^{1}$ Kenji Sasaki, ${ }^{1,2}$ Sinya Aoki, ${ }^{1,2,3}$ Takumi Doi,${ }^{1,4}$ Tetsuo Hatsuda, ${ }^{4,1}$ Yoichi Ikeda, ${ }^{1,5}$ Takashi Inoue, \\ Takumi Iritani, ${ }^{1}$ Noriyoshi Ishii, ${ }^{1,5}$ Takaya Miyamoto, ${ }^{1,2}$ and Hidekatsu Nemura ${ }^{1,5}$ \\ (HAL QCD Collaboration) \\ ${ }^{1}$ RIKEN Nishina Center, RIKEN, Saitama 351-0198, Japan \\ ${ }^{2}$ Center for Gravitational Physics, Yukawa Institute for Theoretical Physics, Kyoto University, Kyoto 606-8502, Japan \\ ${ }^{3}$ Center for Computational Sciences, University of Tsukuba, Ibaraki 305-8571, Japan \\ ${ }^{4}$ RIKEN iTHEMS Program, RIKEN, Saitama 351-0198, Japan \\ ${ }^{5}$ Research Center for Nuclear Physics (RCNP), Osaka University, Osaka 567-0047, Japan \\ ${ }^{6}$ Nihon University, College of Bioresource Sciences, Kanagawa 252-0880, Japan
}

(Received 8 September 2017; revised manuscript received 6 March 2018; published 23 May 2018)

\begin{abstract}
The $\Omega \Omega$ system in the ${ }^{1} S_{0}$ channel (the most strange dibaryon) is studied on the basis of the $(2+1)$ flavor lattice QCD simulations with a large volume $(8.1 \mathrm{fm})^{3}$ and nearly physical pion mass $m_{\pi} \simeq 146 \mathrm{MeV}$ at a lattice spacing of $a \simeq 0.0846 \mathrm{fm}$. We show that lattice QCD data analysis by the HAL QCD method leads to the scattering length $a_{0}=4.6(6)\left({ }_{-0.5}^{+1.2}\right) \mathrm{fm}$, the effective range $r_{\text {eff }}=1.27(3)\left({ }_{-0.03}^{+0.06}\right) \mathrm{fm}$, and the binding energy $B_{\Omega \Omega}=1.6(6)\left({ }_{-0.6}^{+0.7}\right) \mathrm{MeV}$. These results indicate that the $\Omega \Omega$ system has an overall attraction and is located near the unitary regime. Such a system can be best searched experimentally by the pair-momentum correlation in relativistic heavy-ion collisions.
\end{abstract}

DOI: 10.1103/PhysRevLett.120.212001

Introduction.-The dibaryon is defined as a baryon number $B=2$ system (equivalently, a six-quark system) in quantum chromodynamics [1-3]. So far, only one stable dibaryon, the deuteron, has been observed: It is a loosely bound system of the proton and the neutron in spin-triplet and isospin-singlet channel. In recent years, there has been renewed experimental interest in the dibaryons due to exclusive measurements in hadron reactions [4] as well as direct measurement in relativistic heavy-ion collisions [5]. Also, from the theoretical side, $(2+1)$-flavor lattice QCD simulations of the six-quark system in a large box with nearly physical quark masses became possible recently [6]. The main aim of this Letter is to report the first result and physical implication of $\Omega \Omega$, the strangeness $S=-6$ dibaryon ("most strange dibaryon"), in full QCD simulations with the lattice volume $(8.1 \mathrm{fm})^{3}$ and the pion mass $m_{\pi} \simeq 146 \mathrm{MeV}$ at a lattice spacing of $a \simeq 0.0846 \mathrm{fm}$ [7].

Before entering the detailed discussions of our study, we first introduce the reason why such an exotic channel $(S=-6)$ is of interest in QCD. Let us consider octet 8 and

Published by the American Physical Society under the terms of the Creative Commons Attribution 4.0 International license. Further distribution of this work must maintain attribution to the author(s) and the published article's title, journal citation, and DOI. Funded by SCOAP ${ }^{3}$. decuplet $\mathbf{1 0}$ baryons in the flavor-SU(3) classification. All the members of $\mathbf{8}$ are stable under a strong decay. This is why the forces between octet baryons in $\mathbf{8} \otimes \mathbf{8}$ are most relevant in the physics of hypernuclei and of neutron stars. Also, the elusive $H$ dibaryon (a combination of $\Lambda \Lambda, N \Xi$, and $\Sigma \Sigma$ ) is in this representation [8-10] and does not suffer from the Pauli exclusion principle in the flavor-SU(3) limit.

On the other hand, only $\Omega$ in $\mathbf{1 0}$ is stable under a strong decay. Therefore, in the $\mathbf{8} \otimes \mathbf{1 0}$ representation, the most promising candidate of a stable dibaryon is $N \Omega$ [11]. The Pauli exclusion principle does not work in this case, either, so that there is a possibility to have a bound state in the $S$-wave and total-spin 2 channel [12]. Such a system is indeed studied by the two-particle momentum correlation in high-energy heavy-ion collisions both theoretically and experimentally [13].

In the decuplet-decuplet channel, we have

$$
10 \otimes 10=(28 \oplus 27)_{\mathrm{sym}} \oplus\left(35 \oplus \mathbf{1 0}^{*}\right)_{\mathrm{antisym}},
$$

where "sym" and "antisym" stand for the flavor symmetry under the exchange of two baryons. The only possible stable state under a strong decay is the $\Omega \Omega$ system in the symmetric 28 representation. Again, the quark Pauli principle does not operate in this channel [14]. Note that the celebrated $A B C$ resonance $(\Delta \Delta$ in the spin-3 and isospin-0 channel) $[4,15]$ belongs to the antisymmetric 
10* representation, while $\Delta \Delta$ in the spin-0 and isospin-3 channel is in the same multiplet with $\Omega \Omega$.

The $\Omega \Omega$ interaction at low energies has been investigated so far by using phenomenological quark models or by using lattice QCD simulations with heavy quark masses. Very recently, the chiral effective field theory has also been applied to the scattering of the $\Omega$ baryons [16]. In some quark models, a strong attraction is reported $[17,18]$, while other models show a weak repulsion [19,20]. A $(2+1)$ flavor lattice QCD study with $m_{\pi} \simeq 390 \mathrm{MeV}$ by using the finite volume method shows a weak repulsion [21], while a study with $m_{\pi} \simeq 700 \mathrm{MeV}$ by using the HAL QCD method shows a moderate attraction [22]. Under such a controversial situation, it is most important to carry out firstprinciples lattice QCD simulations in a large volume with the pion mass close to the physical point.

HAL QCD method.-In the HAL QCD method [23-26], the observables such as the binding energy and phase shifts are obtained from the equal-time Nambu-Bethe-Salpeter (NBS) wave function $\psi(\boldsymbol{r})$ and associated two-baryon irreducible kernel $U\left(\boldsymbol{r}, \boldsymbol{r}^{\prime}\right)$. The traditional finite volume method with the plateau fitting [27] is challenging in practice for $B=2$ systems in large volumes because of the difficulty in differentiating each scattering state [28-31]. On the other hand, the time-dependent HAL QCD method [25] can avoid such a problem, since all the elastic scattering states are dictated by the same kernel $U\left(\boldsymbol{r}, \boldsymbol{r}^{\prime}\right)$ and there is no need to identify each scattering state in a finite box.

The equal-time NBS wave function $\psi(\boldsymbol{r})$ has the property that its asymptotic behavior at a large distance reproduces the phase shifts, which can be proven from the unitarity of the $S$ matrix in quantum field theories [23]. Moreover, it is related to the following reduced four-point (4-pt) function:

$$
\begin{aligned}
R(\boldsymbol{r}, t>0) & =\langle 0|\Omega(\boldsymbol{r}, t) \Omega(\mathbf{0}, t) \overline{\mathcal{J}}(0)| 0\rangle / e^{-2 m_{\Omega} t} \\
& =\sum_{n} a_{n} \psi_{n}(\boldsymbol{r}) e^{-\left(\delta W_{n}\right) t}+O\left(e^{-\left(\Delta E^{*}\right) t}\right) .
\end{aligned}
$$

Here $\overline{\mathcal{J}}(0)$ is a source operator creating the $(B, S)=$ $(2,-6)$ system at Euclidean time 0 , and $a_{n}$ is the matrix element defined by $\langle n|\overline{\mathcal{J}}(0)| 0\rangle$ with $|n\rangle$ representing the elastic states in a finite volume. The energy is represented as $\delta W_{n}=2 \sqrt{m_{\Omega}^{2}+\boldsymbol{k}_{n}^{2}}-2 m_{\Omega}$ with the $\Omega$ baryon mass $m_{\Omega}$ and the relative momentum $\boldsymbol{k}_{n}$. The typical excitation energy of a single $\Omega$ baryon is denoted by $\Delta E^{*}$, so that the last term in Eq. (1) is exponentially suppressed as long as $t \gg\left(\Delta E^{*}\right)^{-1} \sim \Lambda_{\mathrm{QCD}}^{-1}$ [25] with $\Lambda_{\mathrm{QCD}} \sim 200-300 \mathrm{MeV}$ being the QCD scale parameter. A local interpolating operator for the $\Omega$ baryon has the general form

$$
\Omega(x) \equiv \varepsilon^{a b c}\left[s_{a}^{T}(x) C \gamma_{k} s_{b}(x)\right] s_{c, \alpha}(x),
$$

with $a, b$, and $c$ being color indices, $\gamma_{k}$ being the Dirac matrix, $\alpha$ being the spinor index, and $C \equiv \gamma_{4} \gamma_{2}$ being the charge conjugation. An appropriate spin projection is necessary from this operator to single out a particular spin state as mentioned later.

The reduced 4-pt function $R$ has been shown to satisfy the following master equation [25]:

$$
\left(\frac{\nabla^{2}}{m_{\Omega}}-\frac{\partial}{\partial t}+\frac{1}{4 m_{\Omega}} \frac{\partial^{2}}{\partial t^{2}}\right) R(\boldsymbol{r}, t)=\int d \boldsymbol{r}^{\prime} U\left(\boldsymbol{r}, \boldsymbol{r}^{\prime}\right) R\left(\boldsymbol{r}^{\prime}, t\right),
$$

which is valid as long as $t \gg\left(\Delta E^{*}\right)^{-1}$. We emphasize that we do not need to isolate each scattering state with the energy $\delta W_{n}$, so that only the moderate values of $t$ up to $1.5-2 \mathrm{fm}$ are sufficient for a reliable extraction of the kernel $U$. This is a crucial difference from the finite volume method, which requires $t \gg\left(\delta W_{1}\right)^{-1} \sim 10 \mathrm{fm}$ (for a lattice volume as large as $8 \mathrm{fm}$ ) to identify each $\delta W_{n}$. (See a recent summary [31] and references therein.) At low energies, one can make the derivative expansion with respect to the nonlocality of the kernel [24,32]: $U\left(\boldsymbol{r}, \boldsymbol{r}^{\prime}\right)=V_{0}(r) \delta\left(\boldsymbol{r}-\boldsymbol{r}^{\prime}\right)+$ $\sum_{n=1} V_{2 n}(r) \nabla^{2 n} \delta\left(\boldsymbol{r}-\boldsymbol{r}^{\prime}\right)$. Then, we introduce an "effective" leading-order potential $V(r)$ :

$$
V(r)=R^{-1}(\boldsymbol{r}, t)\left(\frac{\nabla^{2}}{m_{\Omega}}-\frac{\partial}{\partial t}+\frac{1}{4 m_{\Omega}} \frac{\partial^{2}}{\partial t^{2}}\right) R(\boldsymbol{r}, t),
$$

which provides a good leading-order approximation of $U\left(\boldsymbol{r}, \boldsymbol{r}^{\prime}\right)$ to obtain physical observables at low energies, as long as its $t$ dependence is sufficiently small.

Interpolating operator.-The interpolating operator $\Omega_{s_{z}}(x)$ for the $\Omega$ baryon with spin $\frac{3}{2}$ and the $z$ component $s_{z}= \pm \frac{3}{2}, \pm \frac{1}{2}$ can be readily constructed by the appropriate spin projection of the upper two components of Eq. (2) as shown in Ref. [22]. The asymptotic $\Omega \Omega$ system can now be characterized by ${ }^{2 s+1} L_{J}$ with the total spin $(s)$, the orbital angular momentum $(L)$, and the total angular momentum $(J)$. We consider $L=0$ where the maximum attraction is expected at low energies. Then, the Fermi statistics leads $s$ to be even (either $s=0$ or 2). Here we consider an $s=0$ system with the interpolating operator

$$
\begin{aligned}
{[\Omega \Omega]_{0}=} & \frac{1}{2}\left(\Omega_{3 / 2} \Omega_{-3 / 2}-\Omega_{1 / 2} \Omega_{-1 / 2}+\Omega_{-1 / 2} \Omega_{1 / 2}\right. \\
& \left.-\Omega_{-3 / 2} \Omega_{3 / 2}\right) .
\end{aligned}
$$

For $\overline{\mathcal{J}}(0)$, we use the wall-type quark source with the $s=0$ projection given above. With this source, the total momentum of the system is automatically zero. Also, it has good overlap with the scattering states where $|\boldsymbol{r}|$ in Eq. (3) is larger than the typical baryon size. To extract the $L=0$ and $s=0$ states at $t$ on the lattice, we employ Eq. (5) for the sink operator together with the projection to the $A_{1}$ representation of the cubic group, 


$$
P^{\left(A_{1}\right)} R(\boldsymbol{r}, t)=\frac{1}{24} \sum_{i=1}^{24} R\left(\mathcal{R}_{i}[\boldsymbol{r}], t\right),
$$

where $\mathcal{R}_{i}$ is an element of the cubic group acting on the relative distance $\boldsymbol{r}$.

Note here that $R(\boldsymbol{r}, t)$ and $U\left(\boldsymbol{r}, \boldsymbol{r}^{\prime}\right)$ depend on the choice of interpolating operators, while observables calculated from these quantities are independent of the choice thanks to the Nishijima-Zimmermann-Haag theorem [24].

Lattice setup.-By using the $11 \mathrm{PF}$ supercomputer $\mathrm{K}$ at RIKEN Center for Computational Science, $(2+1)$-flavor gauge configurations on the $96^{4}$ lattice are generated with the Iwasaki gauge action at $\beta=1.82$ and nonperturbatively $\mathcal{O}(a)$-improved Wilson quark action with stout smearing. The lattice spacing is $a \simeq 0.0846 \mathrm{fm}\left(a^{-1} \simeq 2.333 \mathrm{GeV}\right)$ [7], and the pion mass, the kaon mass, and the nucleon masses are $m_{\pi} \simeq 146 \mathrm{MeV}, m_{K} \simeq 525 \mathrm{MeV}$, and $m_{N} \simeq 964 \mathrm{MeV}$, respectively. (These masses are higher than the physical values by about $8 \%, 6 \%$, and $3 \%$, respectively, due to slightly larger quark masses at the simulation point.) The lattice size $L a \simeq 8.1 \mathrm{fm}$ is sufficiently large to accommodate two baryons in a box.

We employ the wall quark source with the Coulomb gauge fixing, and the periodic (Dirichlet) boundary condition is used for spatial (temporal) directions. Forward and backward propagations are averaged to reduce the statistical fluctuations. We pick one configuration per each five trajectories and make use of the rotation symmetry and the translational invariance for the source position to increase the statistics. The total statistics in this Letter amounts to 400 configurations $\times 2$ (forward and backward) $\times 4$ rotations $\times 48$ source positions. The quark propagators are obtained by the domain-decomposed solver [33-36], and the correlation functions are calculated using the unified contraction algorithm [37].

The $\Omega$-baryon mass extracted from the effective mass $m_{\mathrm{eff}}(t) \equiv \ln G(t) / G(t+a)$ with $G(t)$ being the baryonic two-point function is $m_{\Omega}=1712 \pm 1 \mathrm{MeV}$ (from the plateau in $t / a=17-22$ ) and $m_{\Omega}=1713 \pm 1 \mathrm{MeV}$ (from $t / a=18-25)$ with the statistical errors. These numbers are about $2 \%$ higher than the physical value of $1672 \mathrm{MeV}$. We take the former number in the following analysis.

Numerical results. - The ${ }^{1} S_{0}$ potential $V(r)$ obtained from Eq. (4) with the lattice measurement of $R(\boldsymbol{r}, t)$ is shown in Fig. 1 for $t / a=16,17$, and 18. Here the Laplacian and the time derivative in Eq. (4) are approximated by the central (symmetric) difference. The statistical errors for $V(r)$ at each $r$ are estimated by the jackknife method with a bin size of 40 configurations. A comparison with the bin size of 20 configurations shows that the bin size dependence is small. The particular region $t / a=$ $17 \pm 1$ in Fig. 1 is chosen to suppress contamination from excited states in the single $\Omega$ propagator at smaller $t$ and simultaneously to avoid large statistical errors at larger $t$. We observe that the potentials at $t / a=16,17$, and 18 are

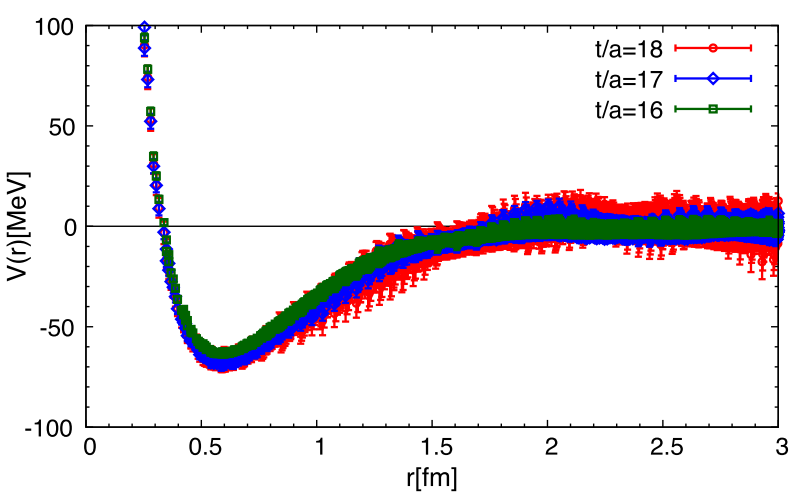

FIG. 1. The $\Omega \Omega$ potential $V(r)$ in the ${ }^{1} S_{0}$ channel at Euclidean time $t / a=16,17$, and 18 .

nearly identical within statistical errors as expected from the time-dependent HAL QCD method [25].

The $\Omega \Omega$ potential $V(r)$ has qualitative features similar to the central potential of the nucleon-nucleon $(N N)$ interaction, i.e., the short-range repulsion and the intermediaterange attraction [6]. There are, however, two quantitative differences: (i) The short-range repulsion is much weaker in the $\Omega \Omega$ case, possibly due to the absence of quark Pauli exclusion effect, and (ii) the attractive part is very shortranged due to the absence of pion exchanges.

For the purpose of converting the potential to physical observables such as the scattering phase shifts and the binding energy, we fit $V(r)$ in Fig. 1 in the range $r=0-6 \mathrm{fm}$ by three Gaussians: $V_{\text {fit }}(r)=\sum_{j=1,2,3} c_{j} \exp \left[-\left(r / d_{j}\right)^{2}\right]$. For example, the uncorrelated fit in the case of $t / a=17$ gives the following parameters: $\left(c_{1}, c_{2}, c_{3}\right)=(914(52)$, $305(44),-112(13))$ in $\mathrm{MeV}$ and $\left(d_{1}, d_{2}, d_{3}\right)=(0.143(5)$, $0.305(29), 0.949(58))$ in $\mathrm{fm}$ with $\chi^{2} /$ d.o.f. $\sim 1.3$. Another functional form such as two Gaussians + (Yukawa function) $)^{2}$ provides an equally good fit, and the results are not affected within errors. The finite volume effect on the potential is expected to be small due to the large lattice size. The naive order estimate of the finite $a$ effect for the physical observables is also small $\left[(\Lambda a)^{2} \leq 1 \%\right]$ thanks to the nonperturbative $O(a)$ improvement, but an explicit confirmation would be desirable in the future.

The $\Omega \Omega$ scattering phase shifts $\delta(k)$ in the ${ }^{1} S_{0}$ channel obtained from $V_{\text {fit }}(r)$ are shown in Fig. 2 for $t / a=16,17$, and 18 as a function of the kinetic energy in the center of mass frame, $E_{\mathrm{CM}}=2 \sqrt{k^{2}+m_{\Omega}^{2}}-2 m_{\Omega}$. The error bands reflect the statistical uncertainty of the potential in Fig. 1. All three cases show that $\delta(0)$ starts from $180^{\circ}$, which indicates the existence of a bound $\Omega \Omega$ system.

The scattering length $a_{0}$ and the effective range $r_{\text {eff }}$ in the ${ }^{1} S_{0}$ channel are extracted from $\delta(k)$ through the effective range expansion, $k \cot \delta(k)=-\left(1 / a_{0}\right)+\frac{1}{2} r_{\text {eff }} k^{2}+\cdots$, with the sign convention of nuclear and atomic physics: 


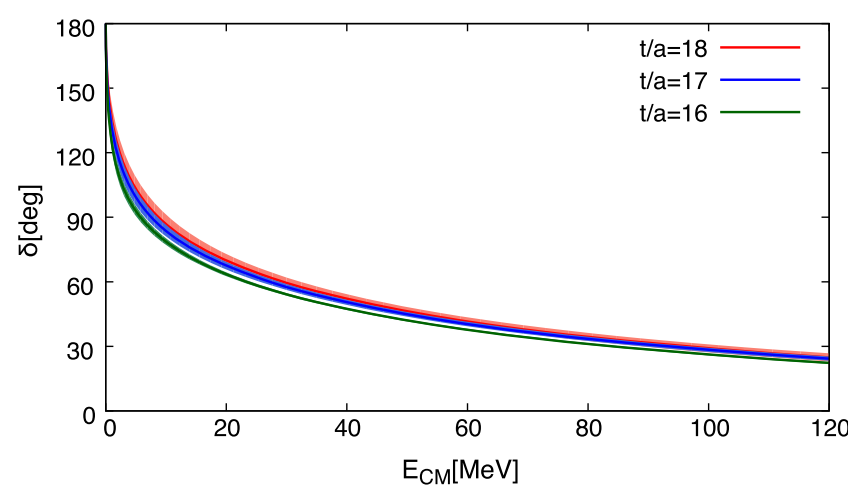

FIG. 2. The $\Omega \Omega$ phase shift $\delta(k)$ in the ${ }^{1} S_{0}$ channel for $t / a=16,17$, and 18 as a function of the center of mass kinetic energy $E_{\mathrm{CM}}=2 \sqrt{k^{2}+m_{\Omega}^{2}}-2 m_{\Omega}$.

$$
\begin{gathered}
a_{0}^{(\Omega \Omega)}=4.6(6)\left(\begin{array}{l}
+1.2 \\
-0.5
\end{array}\right) \mathrm{fm}, \\
r_{\mathrm{eff}}^{(\Omega \Omega)}=1.27(3)\left(\begin{array}{l}
+0.06 \\
-0.03
\end{array}\right) \mathrm{fm} .
\end{gathered}
$$

The central values and the statistical errors in the first parentheses are extracted from $\delta(k)$ at $t / a=17$, and the systematic errors in the second parentheses are estimated from the results at $t / a=16$ and 18 . The origin of this systematic error is the truncation of the higher derivatives of the nonlocal potential as well as the contaminations from inelastic states. To get a feel for the magnitude of these values, we recapitulate here the experimental values of $a_{0}$ and $r_{\text {eff }}$ in the $N N$ systems: $\left(a_{0}, r_{\text {eff }}\right)_{\text {spin-triplet }}=$ $(5.4112(15) \mathrm{fm}, 1.7436(19) \mathrm{fm})$ and $\left(a_{0}, r_{\text {eff }}\right)_{\text {spin-singlet }}=$ $(-23.7148(43) \mathrm{fm}, 2.750(18) \mathrm{fm})$ [38]. There exists no symmetry reason that the scattering parameters in the $N N$ systems and those in the $\Omega \Omega$ system should be similar. Nevertheless, it is remarkable that they are all close to the unitary region where $r_{\text {eff }} / a_{0}$ is substantially smaller than 1 as shown in Fig. 3.

Shown in Fig. 4 are the bound state energy given by the opposite sign of the binding energy, $-B_{\Omega \Omega}$, and the rootmean-square distance $\left(\sqrt{\left\langle r^{2}\right\rangle}\right)$ of the $\Omega \Omega$ bound state obtained from the potential. The blue diamond is taken from the data at $t / a=17$ without the Coulomb repulsion. The blue solid and dashed lines are the statistical error at $t / a=17$ and the systematic error estimated from the data at $t / a=17 \pm 1$, respectively:

$$
B_{\Omega \Omega}^{(\mathrm{QCD})}=1.6(6)\left(\begin{array}{c}
+0.7 \\
-0.6
\end{array}\right) \mathrm{MeV}
$$

As an alternative estimate, the truncation error of the derivative expansion on the binding energy is evaluated perturbatively and is found to be less than $20 \%$ even if the magnitude of the dimensionless next-to-leading-order potential is the same order as that of the effective leadingorder potential. It is an important future subject to determine higher-order potentials explicitly by using the method

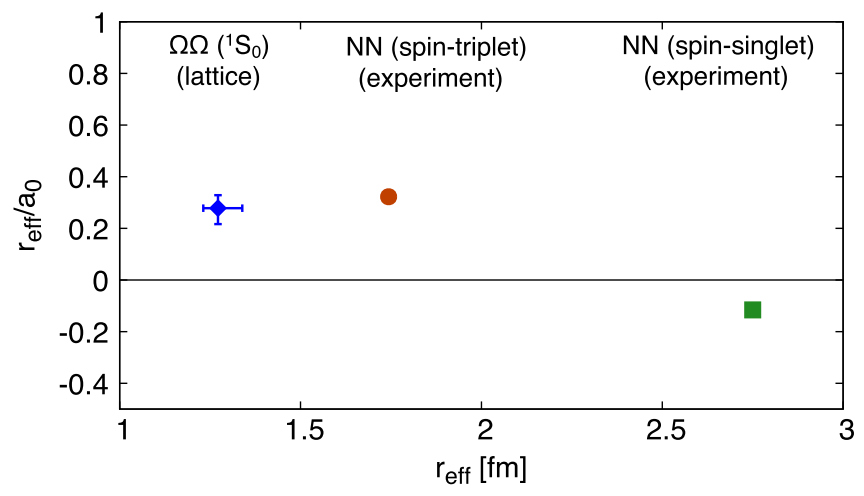

FIG. 3. The dimensionless ratio of the effective range $r_{\text {eff }}$ and the scattering length $a_{0}$ as a function of $r_{\text {eff }}$ for the $\Omega \Omega$ system in the ${ }^{1} S_{0}$ channel as well as for the spin-triplet $N N$ system (the deuteron channel) and for the spin-singlet $N N$ system (the neutron-neutron channel). The error bar for $\Omega \Omega$ is the quadrature of the statistical and systematic errors in Eqs. (7) and (8).

of multiple quark sources [39]. The binding energy is consistent with the value obtained from the general formula for loosely bound states [40] with (7) and (8): $B_{\Omega \Omega}=$ $\left(1 / m_{\Omega} r_{\text {eff }}^{2}\right)\left(1-\sqrt{1-\left(2 r_{\text {eff }} / a_{0}\right)}\right)^{2} \simeq 1.5 \mathrm{MeV}$. Associated with this small binding energy, $\sqrt{\left\langle r^{2}\right\rangle}$ is as large as 3-4 fm, which is consistent with the expectation $\sqrt{\left\langle r^{2}\right\rangle} \sim a_{0}$ for loosely bound states. The Coulomb repulsion can be evaluated by adding $\alpha / r$ with $\alpha=e^{2} / 4 \pi$ to the potential obtained from lattice $\mathrm{QCD}$, i.e., $V^{(\mathrm{QCD}+\text { Coulomb })} \equiv$ $V^{(\mathrm{QCD})}+\alpha / r$. This reduces the above binding energy by a factor of $2: B_{\Omega \Omega}^{(\mathrm{QCD}+\text { Coulomb })}=0.7(5)(5) \mathrm{MeV}$ as shown in Fig. 4 by the red triangle.

It is in order here to remark that there are three energy scales in the present problem: $2 m_{\Omega} \simeq 3400 \mathrm{MeV} \gg$ $|V(r \simeq 0.5 \mathrm{fm})| \sim 50 \mathrm{MeV} \gg B_{\Omega \Omega} \sim 1 \mathrm{MeV}$. Since only

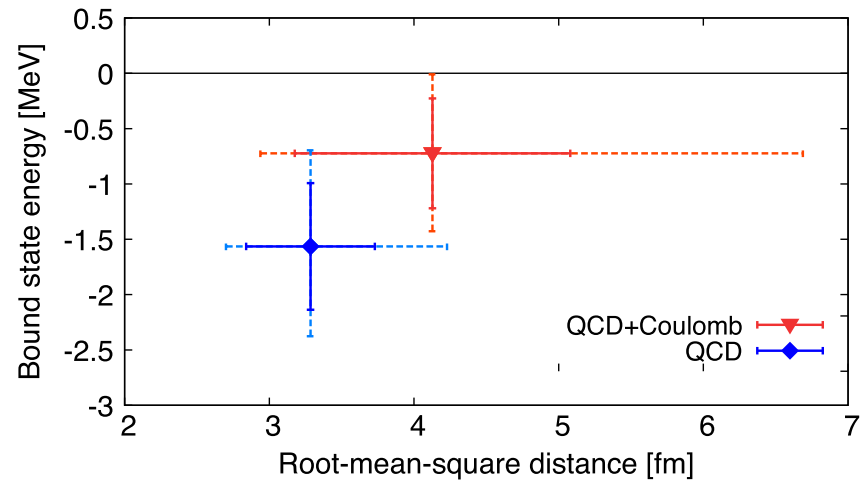

FIG. 4. Bound state energy of the $\Omega \Omega$ system and the rootmean-square distance between $\Omega$ 's obtained from the potential. The filled diamond (triangle) corresponds to the result at $t / a=17$ without (with) the Coulomb repulsion. The statistical errors are shown by the solid lines, while the systematic errors estimated from the difference between the data at $t / a=17$ and those at $t / a=16,18$ are shown by the dashed lines. 
the relative difference between the interacting and noninteracting two- $\Omega$ systems matters, the absolute magnitude of the uncertainty of $2 m_{\Omega}$ is not reflected directly in $V(r)$. This is why we could extract $V(r)$ rather accurately as shown in Fig. 1 despite the large scale difference between $2 m_{\Omega}$ and $V(r)$. Then the small binding energy $B$ as well as the large scattering length $a_{0}$ are the natural consequences of the large cancellation between the long-range attraction and the short-range repulsion of $V(r)$, a situation common in nuclear and atomic physics. Although $V(r)$ is not a direct observable, it provides an important intermediate step to link the QCD scale $(\mathrm{GeV})$ to the nuclear physics scale $(\mathrm{MeV})$, since it is difficult to measure the binding energy directly from lattice QCD using the finite volume method for large lattice volumes and physical quark masses (see the critical review [31]).

Finally, let us estimate the effect of slightly heavy quark masses in our simulation. First of all, the attractive part of the $\Omega \Omega$ potential would be slightly long-ranged at the physical point due to the kaon mass $m_{K}^{\text {(present) }} \simeq 525 \mathrm{MeV} \rightarrow$ $m_{K}^{\text {(phys) }} \simeq 495 \mathrm{MeV}$. On the other hand, the effect of the $\Omega$ mass $m_{\Omega}^{\text {(present) }} \simeq 1712 \mathrm{MeV} \rightarrow m_{\Omega}^{\text {(phys) }} \simeq 1672 \mathrm{MeV}$ would lead to less binding due to the larger kinetic energy. Therefore, a conservative estimate is obtained by keeping the same $V(r)$ in Fig. 1 and adopting $m_{\Omega}^{\text {(phys.) }}$ to calculate the phase shifts and the binding energy. This results in $\left(a_{0}, r_{\text {eff }}\right)=(4.9(8) \mathrm{fm}, 1.29(3) \mathrm{fm})$ and $\left(B_{\Omega \Omega}^{(\mathrm{QCD}+\text { Coulomb })}\right)=$ (1.3(5) MeV, 0.5(5) MeV) for the potential at $t / a=17$. These numbers are well within errors of the results of the present simulation shown in Figs. 3 and 4.

Summary and discussions. - In this Letter, we presented a first realistic calculation on the most strange dibaryon, $\Omega \Omega$, in the ${ }^{1} S_{0}$ channel on the basis of the $(2+1)$-flavor lattice QCD simulations with a large volume and nearly physical quark masses. The scattering length, effective range, and the binding energy obtained by the HAL QCD method strongly indicate that the $\Omega \Omega$ system in the ${ }^{1} S_{0}$ channel has an overall attraction and is located in the vicinity of the unitary regime. From the phenomenological point of view, such a system can be best searched by the measurement of pair-momentum correlation $C(Q)$ with $Q$ being the relative momentum between two baryons produced in relativistic heavy-ion collisions [5]. Experimentally, each $\Omega$ can be identified through a successive weak decay $\Omega^{-} \rightarrow \Lambda+K^{-} \rightarrow p+\pi^{-}+K^{-}$. Note that a large scattering length (not the existence of a bound state) is the important element for $C(Q)$ to have characteristic enhancement at small relative momentum $Q$. Moreover, the effect of the Coulomb interaction can be effectively eliminated by taking a ratio of $C(Q)$ between small and large collision systems [13].

We are currently underway to increase the statistics of the lattice data with the same lattice setup. These results together with the detailed examination of the spectrum analysis in a finite lattice volume and the effective range expansion will be reported.

We thank members of PACS Collaboration for the gauge configuration generation. The lattice QCD calculations have been performed on the K computer at RIKEN, AICS (hp120281, hp130023, hp140209, hp150223, hp150262, hp160211, hp170230), HOKUSAI FX100 computer at RIKEN, Wako (G15023, G16030, G17002), and HA-PACS at University of Tsukuba (14a-20, 15a-30). We thank ILDG/JLDG [41], which serves as an essential infrastructure in this study. We thank the authors of culGT code [42] used for the gauge fixing. This study is supported in part by Grant-in-Aid for Scientific Research on Innovative Areas (No. 2004:20105001, 20105003) and for Scientific Research (No. 25800170 and No. 26400281), SPIRE (Strategic Program for Innovative REsearch), MEXT Grant-in-Aid for Scientific Research (JP15K17667, JP16H03978, JP16K05340), "Priority Issue on Post-K computer" (Elucidation of the Fundamental Laws and Evolution of the Universe), and by Joint Institute for Computational Fundamental Science (JICFuS). S. G. is supported by the Special Postdoctoral Researchers Program of RIKEN. T. D. and T. H. are partially supported by RIKEN iTHES Project and iTHEMS Program. T. H. is grateful to the Aspen Center for Physics, supported in part by NSF Grants No. PHY1066292 and No. PHY1607611. The authors thank C. M. Ko for drawing our attention to the $\Omega \Omega$ system, K. Yazaki for fruitful discussions on the short-range part of baryon-baryon interactions, and Y. Namekawa for his careful reading of the manuscript.

[1] P. J. Mulders, A. T. M. Aerts, and J. J. de Swart, Phys. Rev. D 21, 2653 (1980).

[2] M. Oka, Phys. Rev. D 38, 298 (1988).

[3] A. Gal, Acta Phys. Pol. B 47, 471 (2016).

[4] H. Clement, Prog. Part. Nucl. Phys. 93, 195 (2017).

[5] S. Cho et al. (ExHIC Collaboration), Prog. Part. Nucl. Phys. 95, 279 (2017).

[6] T. Doi et al., Proc. Sci., LATTICE2016 (2017) 110 [arXiv:1702.01600]; H. Nemura et al., Proc. Sci., LATTICE2016 (2017) 101 [arXiv:1702.00734]; K. Sasaki et al., Proc. Sci., LATTICE2016 (2017) 116 [arXiv:1702.06241]; N. Ishii et al., Proc. Sci., LATTICE2016 (2017) 127 [arXiv:1702.03495].

[7] K.-I. Ishikawa et al. (PACS Collaboration), Proc. Sci., LATTICE2015 (2016) 075 [arXiv:1511.09222].

[8] R. L. Jaffe, Phys. Rev. Lett. 38, 195 (1977).

[9] T. Inoue, N. Ishii, S. Aoki, T. Doi, T. Hatsuda, Y. Ikeda, K. Murano, H. Nemura, and K. Sasaki (HAL QCD Collaboration), Phys. Rev. Lett. 106, 162002 (2011).

[10] S. R. Beane et al. (NPLQCD Collaboration), Phys. Rev. Lett. 106, 162001 (2011).

[11] T. Goldman, K. Maltman, G. J. Stephenson, Jr., K. E. Schmidt, and F. Wang, Phys. Rev. Lett. 59, 627 (1987). 
[12] F. Etminan, H. Nemura, S. Aoki, T. Doi, T. Hatsuda, Y. Ikeda, T. Inoue, N. Ishii, K. Murano, and K. Sasaki (HAL QCD Collaboration), Nucl. Phys. A928, 89 (2014).

[13] K. Morita, A. Ohnishi, F. Etminan, and T. Hatsuda, Phys. Rev. C 94, 031901 (2016).

[14] M. Oka, K. Shimizu, and K. Yazaki, Prog. Theor. Phys. Suppl. 137, 1 (2000).

[15] F. Dyson and N. H. Xuong, Phys. Rev. Lett. 13, 815 (1964).

[16] J. Haidenbauer, S. Petschauer, N. Kaiser, U. G. Meiner, and W. Weise, Eur. Phys. J. C 77, 760 (2017).

[17] Z. Y. Zhang, Y. W. Yu, P. N. Shen, L. R. Dai, A. Faessler, and U. Straub, Nucl. Phys. A625, 59 (1997).

[18] Z. Y. Zhang, Y. W. Yu, C. R. Ching, T. H. Ho, and Z.-D. Lu, Phys. Rev. C 61, 065204 (2000).

[19] F. Wang, J. L. Ping, G. H. Wu, L. J. Teng, and T. Goldman, Phys. Rev. C 51, 3411 (1995).

[20] F. Wang, G. H. Wu, L. J. Teng, and T. Goldman, Phys. Rev. Lett. 69, 2901 (1992).

[21] M. I. Buchoff, T. C. Luu, and J. Wasem, Phys. Rev. D 85, 094511 (2012).

[22] M. Yamada, K. Sasaki, S. Aoki, T. Doi, T. Hatsuda, Y. Ikeda, T. Inoue, N. Ishii, K. Murano, and H. Nemura (HAL QCD Collaboration), Prog. Theor. Exp. Phys. 2015, $071 B 01$ (2015).

[23] N. Ishii, S. Aoki, and T. Hatsuda, Phys. Rev. Lett. 99, 022001 (2007).

[24] S. Aoki, T. Hatsuda, and N. Ishii, Prog. Theor. Phys. 123, 89 (2010).

[25] N. Ishii, S. Aoki, T. Doi, T. Hatsuda, Y. Ikeda, T. Inoue, K. Murano, H. Nemura, and K. Sasaki (HAL QCD Collaboration), Phys. Lett. B 712, 437 (2012).

[26] S. Aoki et al. (HAL QCD Collaboration), Prog. Theor. Exp. Phys. 2012, 01A105 (2012).

[27] M. Lüscher, Nucl. Phys. B354, 531 (1991).
[28] G. P. Lepage, in From Actions to Answers: Proceedings of the TASI 1989, edited by T. Degrand and D. Toussaint (World Scientific, Singapore, 1990).

[29] T. Iritani et al., J. High Energy Phys. 10 (2016) 101.

[30] T. Iritani, S. Aoki, T. Doi, T. Hatsuda, Y. Ikeda, T. Inoue, N. Ishii, H. Nemura, and K. Sasaki, Phys. Rev. D 96, 034521 (2017).

[31] S. Aoki, T. Doi, and T. Iritani, EPJ Web Conf. 175, 05006 (2018).

[32] K. Murano, N. Ishii, S. Aoki, and T. Hatsuda, Prog. Theor. Phys. 125, 1225 (2011).

[33] T. Boku et al., Proc. Sci., LATTICE2012 (2012) 188 [arXiv:1210.7398].

[34] M. Terai, K. I. Ishikawa, Y. Sugisaki, K. Minami, F. Shoji, Y. Nakamura, Y. Kuramashi, and M. Yokokawa, in IPSJ Transactions on Advanced Computing Systems (Information Processing Society of Japan, Japan, 2013), Vol. 6, No. 3, pp. 43-57 (in Japanese).

[35] Y. Nakamura, K.-I. Ishikawa, Y. Kuramashi, T. Sakurai, and H. Tadano, Comput. Phys. Commun. 183, 34 (2012).

[36] Y. Osaki and K. I. Ishikawa, Proc. Sci., LATTICE2010 (2010) 036 [arXiv:1011.3318].

[37] T. Doi and M. G. Endres, Comput. Phys. Commun. 184, 117 (2013).

[38] R. W. Hackenburg, Phys. Rev. C 73, 044002 (2006).

[39] T. Iritani (HAL QCD Collaboration) EPJ Web Conf. 175, 05008 (2018).

[40] P. Naidon and S. Endo, Rep. Prog. Phys. 80, 056001 (2017).

[41] http://www.lqcd.org/ildg and http://www.jldg.org.

[42] M. Schröck and H. Vogt, Comput. Phys. Commun. 184, 1907 (2013). 